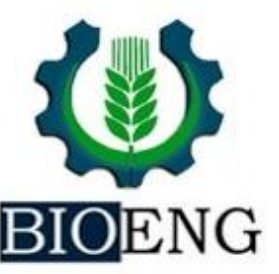

\title{
VARIABILIDADE ESPACIAL E TEMPORAL DO ÍNDICE VEGETAÇÃO MPRI APLICADO ÀS IMAGENS DE GRAMA SÃO CARLOS OBTIDAS POR AERONAVE REMOTAMENTE PILOTADA
}

\author{
L. M. Gonçalves", B. D. S. Barbosa, G. A. e S. Ferraz, D. T. Maciel, \\ H. F. D. Santos
}

\section{UFLA - Universidade Federal de Lavras, Departamento de Engenharia, Lavras, MG, Brasil}

Article history: Received 06 October 2017; Received in revised form 06 November 2017; Accepted 08 November 2017; Available online 27 December 2017.

\begin{abstract}
RESUMO
Imagens de alta resolução obtidas com auxílio de Aeronaves Remotamente Pilotadas (RPA), quando recebem o tratamento adequado podem ser ferramenta útil para a prática da agricultura de precisão, monitorando o crescimento e desenvolvimento da cultura em escala temporal e espacial adequada. Nesse sentido, este trabalho teve por objetivo utilizar imagens obtidas com uma câmera digital acoplada a uma RPA para analisar a variabilidade espacial e temporal do índice de vegetação (IV) MPRI aplicado em uma área de produção grama São Carlos. As imagens foram coletadas durante o período de dezembro de 2016 a março de 2017, utilizando um quadricóptero de voo autônomo, com câmera RGB e altura de voo de $50 \mathrm{~m}$. O processamento das imagens e a aplicação IV MPRI foram realizados com auxílio de um software de geoprocessamento gratuito. Valores médios do MPRI foram gerados para todas as cenas. Foi possível detectar a variabilidade do MPRI em todas a cenas. Um índice de determinação $\left(\mathrm{R}^{2}\right)$ igual a 0,89 foi encontrado devido a correlação entre os valores de MPRI e o tempo após o corte da grama. Pode se inferir por meio dos resultados obtidos que a utilização desta tecnologia tem grande potencial para monitoramento e avaliação das áreas cultivadas com grama.
\end{abstract}

Palavras-chave: Agricultura de Precisão, Axonopus affinis, Geoprocessamento, Sistemas de Aeronaves Não Tripulada (UAS).

\section{SPACE AND TEMPORARY VARIABILITY OF THE INDEX VEGETATION APPLIED TO IMAGES OBTAINED BY A REMOTELY PILOTED AIRCRAFT}

\begin{abstract}
High resolution images obtained with the aid of Remotely Piloted Aircraft (RPA), when properly treated, can be a useful tool for the practice of precision agriculture, monitoring the growth and development of the crop on a suitable temporal and spatial scale. In this sense, this work aimed to use images obtained with a digital camera coupled to a RPA to analyze the spatial and temporal variability of the MPRI vegetation (IV) index applied in a São Carlos grass production area. The images were collected during the period from December 2016 to March 2017, using an autonomous flight quadricopter with RGB camera and flight height of $50 \mathrm{~m}$. The image processing and the MPRI IV application were performed using a free geoprocessing software. Average MPRI values were generated for all scenes. It was possible to detect the variability of MPRI in all scenes. A determination index $\left(\mathrm{R}^{2}\right)$ of 0.89 was found due to the correlation between MPRI values and time after grass cutting. It can be inferred
\end{abstract}

\footnotetext{
*luanna_mendess@yahoo.com.br
} 
from the results obtained that the use of this technology has great potential for monitoring and evaluation of the areas cultivated with grass.

Keywords: Precision Agriculture, Axonopus affinis, Geoprocessing, Unmanned Aircraft Systems (UAS)

\section{INTRODUÇÃO}

O uso de Aeronaves Remotamente Pilotadas (RPA) para a prática da agricultura de precisão, baseado em coletas de imagens e técnicas fotogramétricas para avaliar Índices de Vegetação (IV), representa um recurso rápido, econômico e confiável para o monitoramento de culturas (CANDIAGO et al., 2015), facilitando assim o monitoramento de uma maneira não destrutiva e em tempo real da vegetação (VON BUEREN et al., 2015).

As vantagens e facilidades oferecidas por RPAs em relação às plataformas convencionais utilizadas para imageamento de culturas é devido ao menor custo de aquisição, alta resolução espacial $(\mathrm{cm})$ e temporal das imagens quando comparados a outros sistemas e sensores, como os satélites (TORRES-SÁNCHEZ et al., 2014), e por ser considerada uma ferramenta útil para o monitoramento de precisão no desenvolvimento de culturas, serve como base de dados para apoiar o produtor a otimizar o uso de recursos, realizar o controle de pragas e doenças além de acompanhar a produtividade (BALLESTEROS et al., 2014b).

$\mathrm{O}$ uso do sensoriamento remoto na agricultura e no conceito de agricultura de precisão começou a partir de monitoramento de matéria orgânica no solo através de sensores, sendo que rapidamente esta tecnologia se estendeu a plataformas orbitais, aéreas e montadas em máquinas agrícolas (MULLA, 2013). Ballesteros et al. (2014a), relatam que o uso de RPA com câmera RGB (Red, Green, Blue) acoplada é uma alternativa mais barata e com maior autonomia de uso quando comparada a RPA acoplada à câmera sensível ao espectro do infravermelho (IR- Infra Red). Linhares et al. (2014), ressaltam que o uso de imagens (RGB) obtidas por RPA, são importantes para o monitoramento da safra, em relação a variabilidade espacial e temporal do desenvolvimento vegetativo.

Para avaliar e monitorar o ciclo de desenvolvimento de uma cultura foram desenvolvidos diversos índices de vegetação (IV), estes IV consistem em operações algébricas de valores obtidos de diversas faixas do espectro, nas regiões do visível e do infravermelho próximo principalmente, também denominados como bandas espectrais, e através destes IVs é possível obter informações sobre a cultura (biomassa, dossel, radiação absorvida, teor de clorofila) (SAKAMOTO et al., 2012).

A busca por IV determinados por meio de análises do espectro do visível é constante devido ao baixo custo dos sensores em relação a sensores que captam comprimentos de onda do espectro na região do infravermelho (IR). Alguns autores como Von Bueren et al. (2015), avaliaram sensores embarcados em RPA com captação na região do visível para monitoramento de pastagens para as condições da Nova Zelândia, estes autores concluíram que apesar da câmera com captação espectral na região do visível apresentar desempenho inferior as câmeras com captação multiespectral, os resultados mostram-se satisfatórios.

Yang et al. (2008), em busca de um novo IV que representasse de forma significativa a resposta espectral da vegetação na região do espectro do visível, desenvolveram o MPRI (Modified Photochemical Reflectance Index), este IV avalia a diferença normalizada da resposta espectral na região do verde e do vermelho, com o intuito de realçar a cobertura verde da vegetação.

O mapeamento da cobertura verde do solo com o intuito de estimar o crescimento de uma determinada cultura é 
uma ferramenta que auxilia o produtor na gestão e manejo das áreas cultivadas, principalmente no cultivo de tapete de gramas comerciais. Atualmente na literatura são escassos os estudos que investigam esta possibilidade utilizando RPA com câmeras que captam o espectro da luz na região do visível, embora alguns autores como Rango et al. (2006) e Von Bueren et al. (2015) que investigaram o uso de imagens RPA para monitoramento de pastagem, sem no entanto abordar a

\section{MATERIAL E MÉTODOS}

O estudo foi realizado em uma área cultivada com grama do tipo São Carlos (Axonopus affinis), situado na Fazenda Invernada, no município de Bom SucessoMG, com coordenadas UTM de $23 \mathrm{~K}$ 510525,95 m E, 7662107,43 m S (Figura $1)$.

Foram delimitas regiões em duas áreas de estudo como observado na Figura relação de IVs e estimativa de crescimento da vegetação, e $\mathrm{Li} \& \mathrm{Li}$ (2014) que avaliaram o MPRI no rendimento do trigo no inverno e encontraram uma correlação aceitável com um índice de determinação $\left(R^{2}\right)$ igual a 0,76 .

Diante do exposto, o objetivo deste trabalho foi avaliar a variabilidade temporal e espacial do MPRI em uma área de grama através de imagens obtidas por uma RPA.

1, a área Experimental (AE), onde foram coletadas as imagens durante o período de avaliação, e a área de controle (AC). AC serviu como parâmetro para condições de cobertura vegetal extrema. No qual o IV MPRI, foi determinado como o valor máximo, referente a 270 dias após o corte (DAC), e para servir como referência de valor mínimo igual a 0 DAC.

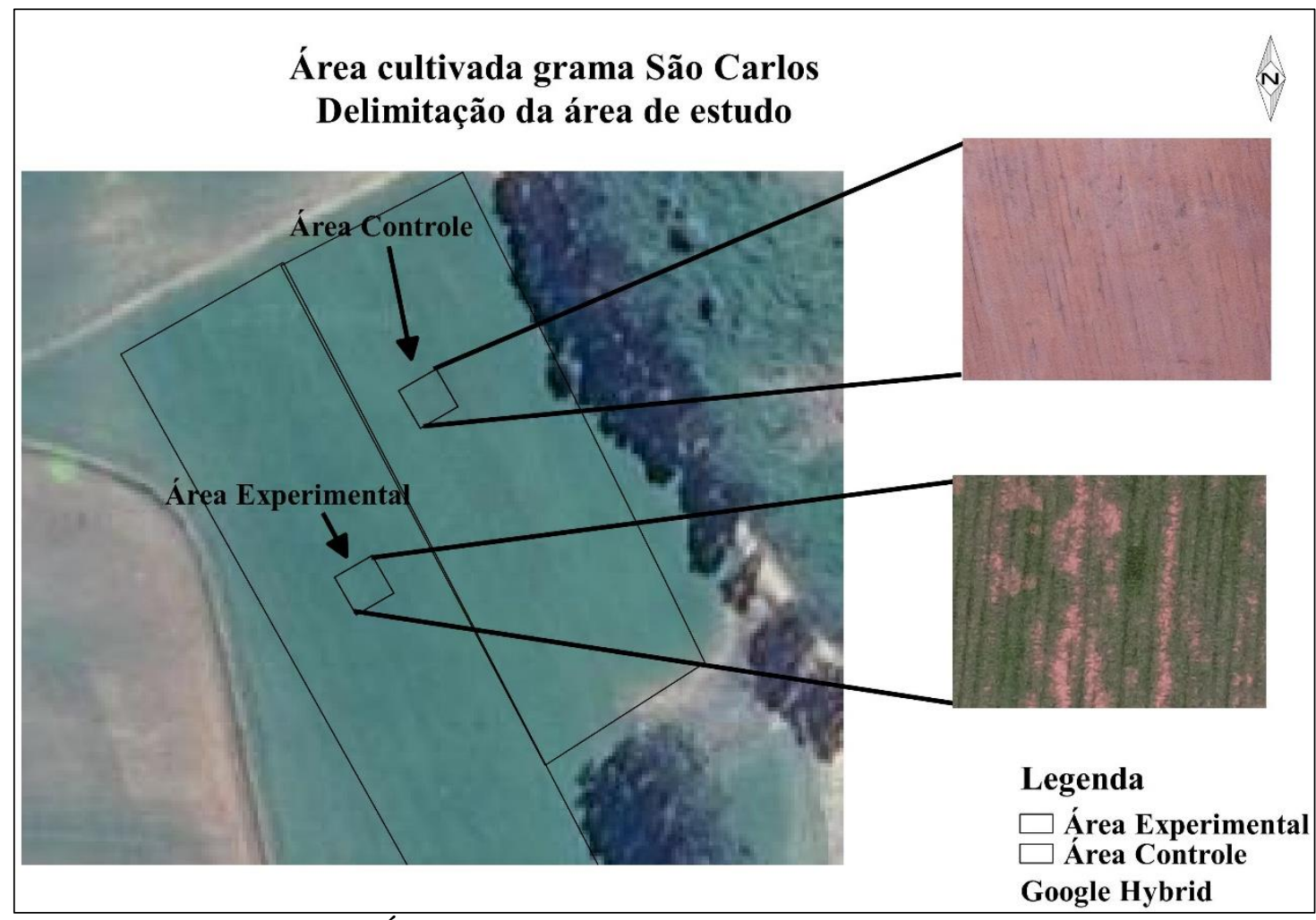

Figura 1. Área de estudo cultivada com grama São Carlos.

As imagens foram adquiridas com o auxílio de um RPA com plataforma de asa rotativa com quatro hélices (quadricóptero), com decolagem e aterrisagem vertical, composto de quatro motores alimentados por uma bateria, com autonomia de voo de até 23 minutos, voo autônomo e gimbal para estabilização da 
câmera durante a obtenção das fotos. Esta RPA possui Sistema de Posicionamento Global (GPS) integrado cujo controle é realizado por meio de um controle remoto. A RPA utilizada possui uma câmera digital acoplada em sua estrutura, com resolução de 12 megapixels em cores verdadeiras (Vermelho-R, Verde-G, Azul-B), e resolução radiométrica de 8 bits, lente 20 $\mathrm{mm}$ com abertura de $\mathrm{f} / 2.8$, com tamanho máximo de imagem de 4000 x 3000 pixels.

As imagens capturadas foram armazenadas em um Cartão SD, e posteriormente exportadas para o software de geoprocessamento para a análise do IV MPRI referente a cada imagem.
Segundo Bater et al. (2011), as imagens de câmeras RGB são fortemente influenciadas por mudanças de iluminação horárias, diárias e sazonais, por isso durante $o$ período de coleta foi padronizado entre as $12 \mathrm{~h}$ e $13 \mathrm{~h}$, visando obter o período de maior luminosidade na área. A altura de voo para a captura das imagens também foi padronizada a $50 \mathrm{~m}$ de altitude em relação ao solo.

A coleta ocorreu durante diferentes estádios de desenvolvimento da grama em relação época em que foi realizado o corte, compreendido entre os meses de dezembro de 2016 a março de 2017, conforme descrito na Tabela 1.

Tabela 1. Datas de coleta das imagens e DAC das áreas de grama São Carlos avaliadas.

\begin{tabular}{ccc}
\hline Área & Data & DAC \\
\hline $\mathrm{AC}$ & $27 / 01 / 2017$ & 0 \\
$\mathrm{AE}$ & $19 / 12 / 2016$ & 11 \\
$\mathrm{AE}$ & $27 / 01 / 2017$ & 50 \\
$\mathrm{AE}$ & $13 / 03 / 2017$ & 95 \\
$\mathrm{AC}$ & $19 / 12 / 2016$ & 270 \\
\hline
\end{tabular}

$\mathrm{AC}=$ Área de Controle, $\mathrm{AE}=$ Área Experimental

O processamento das imagens coletadas nas áreas foi realizado por meio de um software gratuito de geoprocessamento, Qgis versão estável 2.14, no qual utilizou técnicas para quantificar a cobertura vegetal (XIAO \& MOODY, 2005). Para tal, procedeu-se as operações aritméticas com valores obtidos da banda do visível (RBG) baseado no IV MPRI (Modified Photochemical Reflectance Index) proposto por Yang et al. (2008) conforme descrito na (Eq. 1), em que, MPRI = índice de reflexão fotoquímica modificada, decimal; $\mathrm{G}=$ valor de refletância obtidas do espectro na região do verde, decimal; $R=$ valor de refletância

\section{RESULTADOS E DISCUSSÃO}

Na Figura 2 é possível observar as imagens coletadas das áreas de estudo, na região do visível, e seus respectivos obtidas do espectro na região do vermelho, decimal.

$$
M P R I=\frac{(G-R)}{(G+R)} \quad \text { Eq.1 }
$$

Posteriormente fez-se a confecção do layout dos mapas no mesmo software de geoprocessamento.

Após o processamento das imagens coletadas durante $o$ período, foram extraídos valores do MPRI em todos os pixels das imagens, e através de estatística por zona, foram avaliados a média, a mediana, o desvio padrão, o valor mínimo e máximo dos pixels do mapa do IV MPRI da grama São Carlos em diferentes idades de cultivo, possibilitando assim uma análise de regressão linear em função do DAC.

períodos de análise conforme descrito na Tabela 1. 


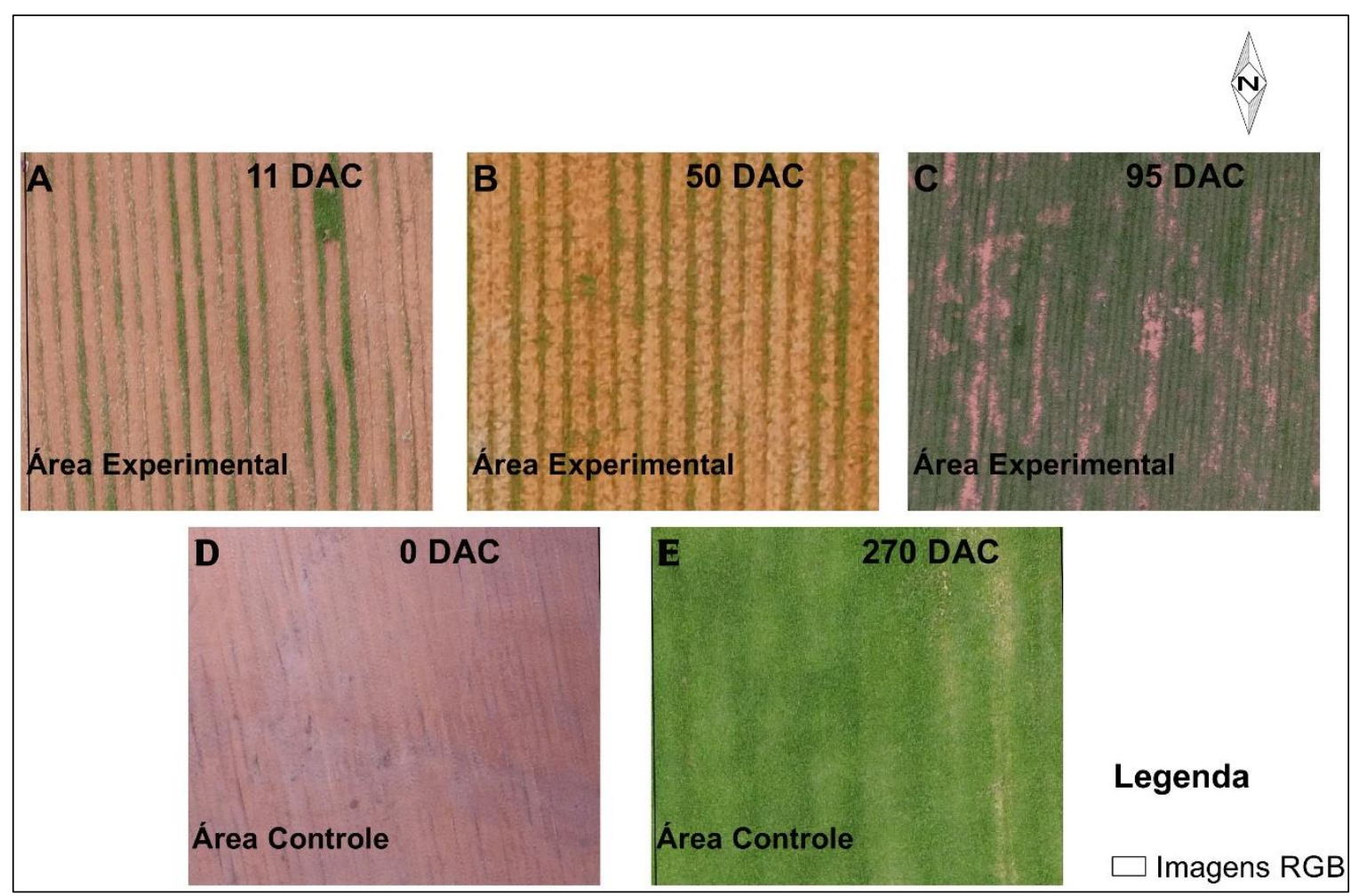

Figura 2. Imagens na região do visível (RGB) das áreas de estudo: A-Área Experimental com 11 DAC, B- Área Experimental com 50 DAC, C- Área Experimental com 95 DAC, D- Área Controle com 0 DAC e E- Área Controle com 270 DAC.

Nestas imagens é possível observar a $\mathrm{AC}$ (Figura 2E), que representa a área em ponto de colheita, com solo totalmente coberto pela grama $(100 \%$ de cobertura verde) representando neste estudo o valor máximo de MPRI que possa ser extraído de um pixel, com período de 270 DAC. A área $\mathrm{AC}$, representada pela Figura 2D, permite a observação do solo praticamente sem cobertura verde, sendo este estágio de desenvolvimento igual a um período de 0 DAC. Os valores de MPRI extraídos destas imagens representam os valores extremos de cobertura verde na área.

$\mathrm{A}$ área experimental (AE) representada pelas Figuras $2 \mathrm{~A}, 2 \mathrm{~B}$ e $2 \mathrm{C}$ corresponde a mesma área, porém com DAC distintos, sendo possível acompanhar o desenvolvimento destas áreas ao longo período nos quais foram obtidas as imagens.

A coleta das imagens por meio de câmeras digitais geralmente é realizada nas configurações automáticas podendo ser satisfatórias, porém com resolução radiométrica baixa, esta resolução pode interferir nas estimativas quantitativas de parâmetros da superfície. Lebourgeois et al. (2008) mostraram que um processo simples de correção radiométrica na qual um fluxo de processamento de imagem realizado permite a melhoria desta resolução e um melhor acompanhamento das culturas usando câmeras digitais comerciais a bordo de RPA.

Neste estudo não foi realizado correções radiométricas, no entanto, este auto ajuste da câmera não interferiu de maneira significativa na obtenção do IV MPRI, sendo possível observar a variabilidade da vegetação de acordo com o estágio de desenvolvimento dos tapetes de grama em uma sequência lógica.

Após o pré-processamento, foi possível calcular o MPRI, e obter o comportamento do mesmo em função de DAC, conforme observa-se na Figura 3. De acordo com Delegido et al. (2013) os IV salientam características da cobertura vegetal e facilita a obtenção de informações relevantes de imagens digitais. 


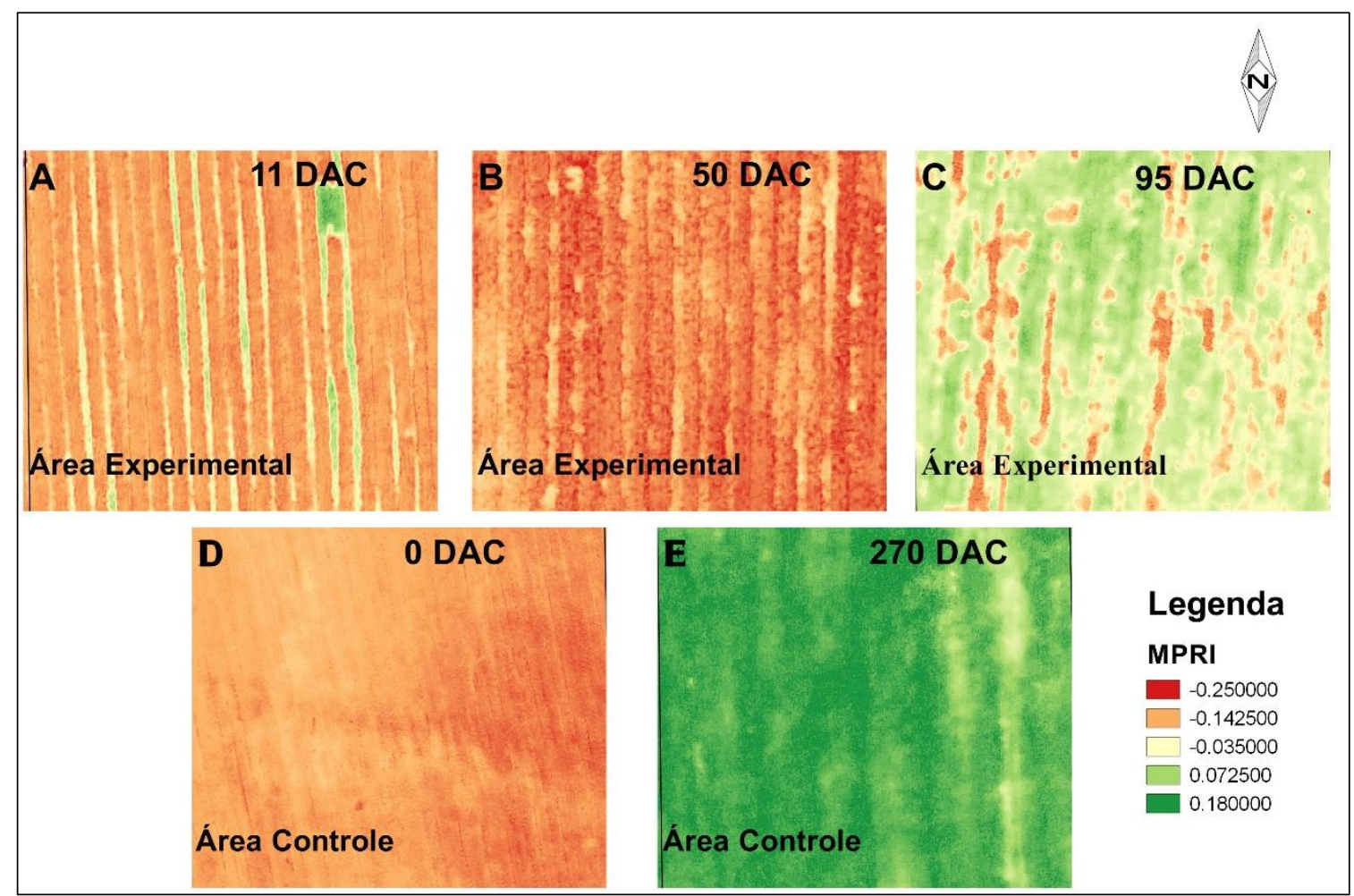

Figura 3. Imagens da área de estudo após o processamento para obtenção do IV MPRI. A-Área Experimental com 11 DAC, B- Área Experimental com 50 DAC, C- Área Experimental com 95 DAC, D- Área Controle com 0 DAC e E- Área Controle com 270 DAC.

Durante o período de coleta das imagens em campo, pode-se perceber a variabilidade temporal e espacial no crescimento da grama São Carlos (Figura 3), onde nota-se a variabilidade dos valores de MPRI a medida que os DAC aumentam. Nota-se também ao analisar as Figuras 3A, $3 \mathrm{~B}$ e $3 \mathrm{C}$, que o crescimento da grama não ocorre de uma maneira desuniforme na área, este comportamento pode estar associado a diversos fatores como o solo e a distribuição espacial de suas propriedades.

Uma análise estatística foi realizada a fim de verificar a variabilidade temporal dos valores de MPRI na área, conforme valores descritos na Tabela 2.

Tabela 2. Estatística descritiva dos pixels das imagens do IV MPRI.

\begin{tabular}{ccccccc}
\hline Área & DAC & Méd. & Med. & D.P. & Mín. & Máx. \\
\hline AC & 0 & $-0,15$ & $-0,15$ & 0,02 & $-0,64$ & $-0,01$ \\
AE & 11 & $-0,13$ & $-0,14$ & 0,04 & $-0,33$ & 0,18 \\
AE & 50 & $-0,17$ & $-0,18$ & 0,04 & $-0,86$ & 0,03 \\
AE & 95 & $-0,03$ & $-0,01$ & 0,06 & $-0,48$ & 0,19 \\
AC & 270 & 0,12 & 0,12 & 0,03 & $-0,21$ & 0,33 \\
\hline
\end{tabular}

Analisando a Figura 3D e Tabela 2, nota-se que a área com maior porção de solo exposto, referente à 0 DAC, o valor médio encontrado de MPRI para esta cena é de $-0,15$. Esta cena ilustra a situação de solo praticamente desprovido de cobertura verde, apresentando valores de IV MPRI variando entre $-0,08$ a $-0,18$, estes valores negativos para refletância do IV MPRI.
O estudo do MPRI tem potencial promissor, segundo Linhares et al. (2017) concluíram que a resposta espectral do MPRI para vegetação e solo tem alta correlação com o NDVI. Demarchi et al. (2011) analisando a variação temporal do uso de solo encontrou valores para solo exposto concentraram-se entre as classes 
de NDVI -0,1 a 0,2, o que se assemelha aos valores encontrados neste estudo.

Ainda na Figura 3D, a região sudoeste apresentou valores na faixa de 0,25 , enquanto na maioria da área os valores foram na faixa de $-0,14$. Esta região representa solo totalmente exposto, entretanto observa-se também nesta região maiores valores do IV, isso se justifica devido à sombra de nuvem que cobriu esta região no momento da coleta da foto e interferiu na iluminação favorecendo este resultado. De acordo com Gitelson et al. (2002), as informações derivadas de um IV geralmente são menos sensíveis à iluminação e outros fatores que afetam a reflectância dos objetos. Nas áreas representadas nas Figuras 3A, 3B e 3C, observa-se a evolução e $\mathrm{o}$ aumento significativo dos valores de MPRI, referente ao aumento da cobertura verde sobre o solo.

Após 11 DAC, o valor médio de MPRI é de -0,13 (Figura 3A e Tabela 2), após 50 DAC este valor não tem o comportamento que era esperado de elevar à medida que a cobertura verde aumenta na área (Figura 3B e Tabela 2), registrando um valor médio de $-0,17$, aos 95 DAC (FIGURA 3C) o valor médio de MPRI computado foi de $-0,03$ (Tabela 2).

O comportamento não esperado do valor encontrado com 50 DAC pode ser explicado devido ao conceito da mistura espectral abordada por Linhares et al. (2014), que, ao analisar o comportamento do MPRI no desenvolvimento da soja, encontrou um valor médio baixo de MPRI para o estádio de desenvolvimento inicial da cultura, no qual o valor de um pixel pode ser contaminado por outros objetos como o solo, já que a soja neste estádio não forma dossel significativo. Este estádio da grama também é um estádio onde tem solo exposto, resto de palhada e grama (Figura 3B) em crescimento podendo ter causado esta mistura espectral favorecendo este baixo valor de IV.

Outro fator que pode estar associado a este comportamento dos valores de MPRI observados é que, durante o período de estudo a cobertura verde da grama na área ainda não foi suficiente para representar mudanças significativas nos valores de MPRI, ressaltando assim, a importância de um maior período de análise para esta cultura, já que não foi encontrado na literatura dados consistentes que possam fornecer um maior embasamento para a discussão sobre o comportamento observado dos valores de MPRI, principalmente para a cultura em estudo.

$\mathrm{Na}$ Figura 3B, percebe-se uma predominância de valores na faixa do 0,25 , entretanto há ainda algumas linhas de falhas contendo grama que não foi colhida, intencionalmente para que haja a rebrota da mesma, apresentando valores de 0,18 , e outras linhas com valores de $-0,035$ que seria a resposta da palhada deixada no solo após a colheita.

Na Figura 3C com 95 DAC, a grama já encontrava-se em crescimento, nesta cena é destacado valores de MPRI igual a 0,00725. Observa-se na imagem que há ainda solo exposto, devido aos valores de MPRI igual -0,25, e outros valores intermediários que se destacam na cena como -0,035 e -0,143. Esta cena mostra a desuniformidade do crescimento, podendo ser ferramenta para obter os locais com tais falhas e identificar a causa raiz do problema em campo.

Ainda na região sudoeste da Figura 3C são encontrados valores na faixa do 0.143 , enquanto na maioria da área os valores de MPRI ficaram entre 0,00725 a 0.18. Ademais, pode-se observar nesta cena que existem pontos cujo MPRI estava mais baixo, quase representando o solo exposto de -0,25. Assim, estas imagens podem ser úteis na identificação de possíveis falhas que possam vir ocorrer ao longo do período de cultivo.

Observa-se nas Figuras $3 \mathrm{~B}$ e $3 \mathrm{C}$ que há regiões onde o crescimento vegetativo da grama apresenta falhas, estas podem estar relacionada a diversos fatores, como por exemplo: i) declividade da área, que ocasiona um escoamento de água; ii) sistema de irrigação, ser do tipo autopropelido, no qual sua eficiência é altamente influenciada por fatores 
atmosféricos como o vento, no qual vale ressaltar que nesta área durante o período e coleta a velocidade era considerável, pois teve situações que a decolagem da RPA teve de ser adiada por algum tempo até que a velocidade do vento fosse reduzida a uma condição favorável ao voo da aeronave. Tais falhas carecem de mais estudos, e maior período de monitoramento, para que se possa apontar qual fator tem maior influência nestas descontinuidades.

No período de 270 DAC o valor médio do MPRI foi igual a 0,12 (Figura 3E e Tabela 2). Este cenário representa a situação onde a cobertura verde no solo tem os maiores valores de MPRI por pixel amostrado. Nesta mesma imagem nota-se na borda superior direita da AC menores valores de MPRI igual a 0,063, já na inferior esquerda teve valores maiores para o índice. Estes valores demostram que o crescimento desuniforme das áreas, podendo acarretar alterações no cronograma de colheita.

Apesar de ter uma predominância de cores, no caso verde, observa-se um desenvolvimento irregular, na qual os valores do IV variou de 0,18 a $-0,0035$ indicando a existência de variabilidade espacial na área. Valores próximos foram encontrados nos estudos de Linhares et al. (2014), no qual valores médios de MPRI para o estádio R2 de desenvolvimento da soja, que corresponde ao estádio reprodutivo da cultura contendo o maior vigor vegetativo, foram de 0,2752 corroborando com os resultados encontrados, onde quanto maior $\mathrm{o}$ teor vegetativo maior o valor do índice.

Os dados descritos na Figura 4 demonstram que em média, os valores de MPRI das cenas avaliadas da área crescem linearmente ao longo do tempo, exceto para a área com 50 DAC que apresentou um valor menor na sequência.

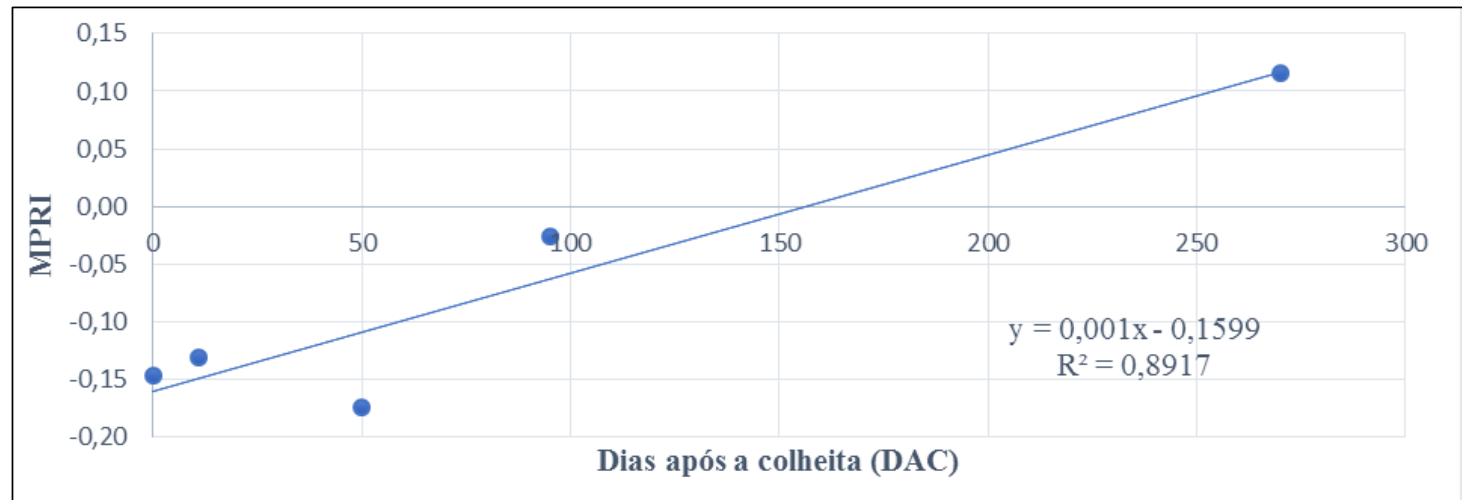

Figura 4. Gráfico dos valores médios do MPRI dos pixels das imagens em função do DAC.

Apesar deste ponto (50 DAC) não ter se comportado de maneira linear, o coeficiente de determinação encontrado ainda foi de $89,17 \%$. Entretanto ressalta-se a necessidade de continuar $o$ acompanhamento da área e obter dados que ratifiquem estes resultados. De acordo com os dados da Figura 4 foi possível determinar uma equação linear do MPRI em função do DAC (Eq.2), em que, MPRI $=$ índice de reflexão fotoquímica modificada, decimal; $\mathrm{DAC}=$ dias após $\mathrm{o}$ corte.

$$
M P R I=0,001 D A C-0,1599 \text { Eq. } 2
$$

Na literatura ainda há poucos estudos utilizando o MPRI e sua variabilidade espacial, principalmente em gramas. Atualmente o IV mais utilizado e abordado na literatura que correlaciona o desenvolvimento de culturas é o NDVI, que é determinado em função da radiação eletromagnética emitida pela vegetação na porção do espectro referente ao IR. Ballasteros et al. (2014b), relatam que o NDVI é passível de ser substituído por outras técnicas de imageamento e processamento de imagens e outros índices de vegetação que se baseiam nas informações obtidas através das bandas do espectro do visível. 


\section{CONCLUSÕES}

Foi possível detectar a variabilidade espacial e temporal do índice de vegetação MPRI aplicado nas imagens de grama São Carlos por meio do uso de uma aeronave remotamente pilotada (RPA) com câmera

\section{AGRADECIMENTOS}

A Itograss Agrícola Ltda por ceder a área de estudo. A UFLA e ao PPGEA pelo

\section{REFERÊNCIAS}

BALLESTEROS, R.; ORTEGA， J. F.; MORENO, M. A. Applications of georeferenced high-resolution images obtained with unmanned aerial vehicles. Part I: Description of image acquisition and processing. Precision Agriculture, v. 15 , n. 6, p. 579-592, 2014a.

BALLESTEROS, R.; HERNÁNDEZ, D.; ORTEGA, J. F.; MORENO, M. A. Applications of georeferenced highresolution images obtained with unmanned aerial vehicles. Part II: application to maize and onion crops of a semi-arid region in Spain. Precision Agriculture, v. 15, n. 6, p. 593-614, 2014b.

BATER, C. W.; COOPS, N. C.; WULDER, M. A.; HILKER, T.; NIELSEN, S. E.; MCDERMID, G.; STENHOUSE, G. B. Using digital timelapse cameras to monitor species-specific understorey and overstorey phenology in support of wildlife habitat assessment. Environmental monitoring and assessment, v. 180, n. 1, p. 1-13, 2011.

CANDIAGO, S.; REMONDINO F.; GIGLIO, de M.; DUBBINI, M.; GATTELI, M. Evaluating multispectral images and vegetation indices for precision farming applications from UAV images. Remote Sensing, Basel, v. 7, n. 4, p. 40264047, 2015.

DELEGIDO, J.; VERRELST, J.; MEZA, C. M.; RIVERA, J. P.; ALONSO, L.; digital embarcada. Este conjunto de equipamentos e técnicas apresentou um potencial auxilio no controle e manejo em áreas de cultivo de grama.

apoio ao estudo. A CAPES pela bolsa de estudo dos autores, e ao SISU/MEC/PET.

MORENO, J. A red-edge spectral index for remote sensing estimation of green LAI over agroecosystems. European Journal of Agronomy, v. 46, p. 42-52, 2013.

DEMARCHI, J. C.; PIROLI, E. L.; ZIMBACK, C. R. L. Analise temporal do uso do solo e comparação entre os índices de vegetação NDVI e SAVI no município de Santa Cruz do Rio Pardo-SP usando imagens LANDSAT-5. Raega- O Espaço Geográfico em Análise, v. 21, 2011.

GITELSON, A. A.; KAUFMAN, Y. J.; STARK, R.; RUNDQUIST, D. Novel algorithms for remote estimation of vegetation fraction. Remote sensing of Environment, v. 80, n. 1, p. 76-87, 2002.

LEBOURGEOIS, V.; BÉGUÉ, A.; LABBÉ, S.; MALLAVAN, B.; PRÉVOT, L.; ROUX, B. Can commercial digital cameras be used as multispectral sensors? A crop monitoring test. Sensors, v. 8, n. 11, p. 7300-7322, 2008.

LI, S. \& LI, M. Yield estimation of winter wheat in early growth periods by vehicleborne ground-based remote sensing system. Transactions of the Chinese Society of Agricultural Engineering, 30(3), 120-127. 2014.

LINHARES, M. M. A.; AMARAL, B. A. S.; COSTA, A. P.; ROCHA, N. C. C.; CÂNDIDO, L. G. Vant's aplicados ao monitoramento da evolução vegetativa de 
culturas agrícolas. In: CONGRESSO BRRASILEIRO DE AGRICULTURA DE PRECISÃO, 2014, São Pedro. Anais...São Pedro, 2014. Disponível em: $<$ http://www.sbea.org.br/conbap/2014/trab alhos/R0062-2.PDF>. Acesso em: 15 ago 2017.

LINHARES, M. M. A.; ROCHA, N. C. C.; AMARAL, B. A. S. Análise do índice MPRI como indicador vegetativo através da correlação do mesmo com o índice NDVI. In: SIMPÓSIO BRASILEIRO DE SENSORIAMENTO REMOTO, Foz do Iguaçu, PR, Brasil. Disponível em: < http://www.dsr.inpe.br/sbsr2013/files/p119 0.pdf >. Acesso em: 25 mar. 2017.

MULLA, D. J. Twenty five years of remote sensing in precision agriculture: Key advances and remaining knowledge gaps. Biosystems engineering, v. 114, n. 4, p. 358-371, 2013.

RANGO, A.; LALIBERTE, A.; STEELE, C.; HERRICK, J. E.; BESTELMEYER, B.; SCHMUGGE, T.; JENKINS, V. Using unmanned aerial vehicles for rangelands: current applications and future potentials. Environmental Practice, v. 8, n. 03, p. 159-168, 2006.

SAKAMOTO, T.; GITELSON, A. A.; NGUY-ROBERTSON,

L.;ARKEBAUER, T. J.; WARDLOW, B. D.; SUYKER, A. E.; VERMA, S. B.; SHIBAYAMA, M. An alternative method using digital cameras for continuous monitoring of crop status. Agricultural and Forest Meteorology, Amsterdam, v. 154, p. 113-126, 2012.

TORRES-SÁNCHEZ, J.; PENÃ，J. M.; CASTRO, A. I. D.; LÓPEZ-GRANADOS, F. Multi-temporal mapping of the vegetation fraction in early-season wheat fields using images from UAV. Computers and Electronics in Agriculture, Amsterdam, v. 103, p. 104113, 2014.
VON BUEREN, S. K.; BURKART, A.; HUENI, A.; RASCHER, A.; TUOHY, M. P.; YULE, I. J. Deploying four optical UAV-based sensors over grassland: challenges and limitations. Biogeosciences, Göttingen, v. 12, n. 1, p. 163, 2015.

XIAO, J. \& MOODY, A. A comparison of methods for estimating fractional green vegetation cover within a desert-to-upland transition zone in central New Mexico, USA. Remote Sensing of Environment, Amsterdam, v. 98, n. 2, p. 237-250, 2005.

YANG, Z.; WILLIS, P.; MUELLER, R. Impact of Band-Ratio Enhanced AWIFS Image to Crop Classification Accuracy. Disponível em: <https://www.asprs.org/a/publications/proc eedings/pecora17/0041.pdf $>$. Acesso em: 15 ago 2017. 